\title{
Clinical application value of serum neutrophil gelatinase-associated lipocalin in neonatal sepsis
}

\author{
Qun Cai ${ }^{1}$, Xiaoqun Zhang ${ }^{1}$, Liyuan Shen ${ }^{1}$, Ting Wang ${ }^{2}$ \\ ${ }^{1}$ Department of Pediatrics, Affiliated Hospital of Nantong University, Nantong, China; ${ }^{2}$ Department of Emergency, Affiliated Hospital of Nantong \\ University, Nantong, China \\ Contributions: (I) Conception and design: Q Cai, T Wang; (II) Administrative support: Q Cai, X Zhang, T Wang; (III) Provision of study materials \\ or patients: Q Cai, X Zhang, L Shen; (IV) Collection and assembly of data: Q Cai, L Shen, T Wang; (V) Data analysis and interpretation: Q Cai, X \\ Zhang, T Wang; (VI) Manuscript writing: All authors; (VII) Final approval of manuscript: All authors. \\ Correspondence to: Ting Wang. Department of Emergency, Affiliated Hospital of Nantong University, 20 Xisi Road, Chongchuan District, Nantong \\ 226001, China. Email: nt_wangting@163.com.
}

Background: To explore the value of serum neutrophil gelatinase-associated lipocalin (sNGAL) in the diagnosis and follow up of neonatal late-onset sepsis.

Methods: A total of 69 infants were enrolled in this prospective study, including 49 infants of late-onset neonatal sepsis in the observation group, and 20 infants without infection serving as the control group. The sNGAL, C-reactive protein (CRP), and procalcitonin (PCT) concentrations were determined in both groups and compared at different time points. A receiver operating characteristic (ROC) curve was drawn to evaluate the values of the 3 parameters in the forecast of neonatal late-onset sepsis.

Results: The levels of sNGAL, CRP, and PCT were all increased obviously $(\mathrm{P}<0.05)$ in the observation group on the first and second day following onset, compared to the control group. The sNGAL level was associated with the time of treatment. Surprisingly, the sNGAL level started to drop in the observation group with effective treatment on the 7th day following onset. A correlation was found between the concentration of sNGAL and inflammatory markers, such as CRP and PCT, on the first day. The area under the ROC curve (AUC) for sNGAL, CRP, and PCT was: 0.964, 0.925, and 0.94, respectively.

Conclusions: Increased sNGAL levels could reflect the inflammatory status in the acute stage of neonatal sepsis. When combined with other sepsis markers, such as CRP and PCT, the sNGAL is a useful marker in the rapid diagnosis and follow up of neonatal sepsis.

Keywords: Serum neutrophil gelatin-related lipid delivery protein; C-reactive protein (CRP); procalcitonin; neonatal sepsis

Submitted Nov 12, 2021. Accepted for publication Jan 13, 2022.

doi: $10.21037 / \mathrm{tp}-21-587$

View this article at: https://dx.doi.org/10.21037/tp-21-587

\section{Introduction}

Neonatal sepsis is the most common cause of morbidity and mortality in newborns. The early clinic manifestations are non-specific, but the condition can rapidly deteriorate into shock, disseminated intravascular coagulation (DIC), and multiple organ dysfunction syndrome (MODS) $(1,2)$. Most recently, a study estimated that the mortality of neonatal sepsis is about $11-19 \%$, which will bring a heavy burden to the family and society (3). Therefore, early detection, accurate diagnosis, and effective antimicrobial therapy for neonatal sepsis have always been important and clinically challenging $(4,5)$. The gold standard for diagnosis of neonatal sepsis is isolation of organism in blood culture, but culture of the organism is lengthy, the cultivation conditions are strict, and the sensitivity is low $(6,7)$. Thus, the blood culture does not facilitate early diagnosis (8). At present, biomarkers for diagnosis of neonatal sepsis include C-reactive protein (CRP), 
procalcitonin (PCT), white blood cell count (WBC), platelet count, and so on, but each of them has its limitation (6). CRP is a pentameric structure protein and is an acute phase reactant protein, which is the most commonly used laboratory tests in the diagnosis of neonatal sepsis. PCT is also an acute phase reactant protein and is peptide prohormone of calcitonin. Currently, researchers are still searching for more sensitive and specific biomarkers, such as CD64 (9), serum neutrophil gelatinase-associated lipocalin (sNGAL) (10), both of which reflect the activation of neutrophils after infectious stimulation. NGAL is a small circulating protein that is highly modulated in a wide variety of pathological situations, making it a useful biomarker of various disease states. NGAL is a lipocalin superfamily member, of which elevations in both the urine and plasma concentration occur at an early stage of acute kidney injury (AKI) of sepsis. So far, little research has been conducted regarding the value of sNGAL in neonatal sepsis. In this study, we observed the dynamic changes of sNGAL in late-onset neonatal sepsis, and compared them with the infectious biomarkers of CRP and PCT. We present the following article in accordance with the STARD reporting checklist (available at https:// tp.amegroups.com/article/view/10.21037/tp-21-587/rc).

\section{Methods}

\section{Participants}

Patients recruited to this study were from the neonatal intensive care unit (NICU) of the Affiliated Hospital of Nantong University between March 2019 and March 2020, and met the inclusion criteria for late-onset sepsis according to the Expert Consensus on the Diagnosis and Treatment of Neonatal Sepsis (2019 edition) (11). We included 49 patients in the observation group. We also randomly included a control group comprising 20 non-sepsis newborns. Data were collected on admission for gender, gestational age, the onset of day age, birth weight, Apgar score, the level of creatinine, platelet count, and so on. The gestational age of the 2 groups was $\geq 37$ weeks. The exclusion criteria were as follows: congenital heart disease, congenital renal disease, and congenital immunodeficiency. As per instructions of the Ethics Committee of the Affiliated Hospital of Nantong University, informed consent was provided by the guardians of patients before commencement of the study. The study was approved by the Ethics Committee of the Affiliated Hospital of Nantong University (2021-K141-01), and was conducted in accordance with the Declaration of Helsinki (as revised in 2013).

\section{Data collection}

All participants were treated according to the routine management of neonatal diseases. Blood culture and/ or cerebrospinal fluid culture of the observation group were conducted before the antibiotic administration. Blood samples were obtained on day 1 , day 2, and day 7 of NICU admission. Blood cell counts, CRP, PCT, and creatinine determinations were made promptly by the hospital's central laboratory. Samples for sNGAL analysis were made by the laboratory of nephrology with double antibody sandwich immunochromatography (Beijing Hot View Biotechnology Co., Ltd., Beijing, China), based on documentation provided by the manufacturer. The normal range for sNGAL is $<145 \mathrm{ng} / \mathrm{mL}$ (10).

\section{Statistical analysis}

All statistical analyses were performed using the software SPSS 25.0 (IBM Corp., Chicago, IL, USA). The ShapiroWilk test was used to analyze the distribution of data. Measurement data with normal distribution were presented as mean \pm standard deviation, and compared using $t$-test for comparison between groups. Abnormally distributed measurement data were presented as median and quaternary, and compared using Mann-Whitney U test. Repeated test data analysis of variance (ANOVA) was used for repeated measurements at the same time point. The rates describing the count data were compared using Pearson's chi-square test and Fisher's exact test. Pearson correlation analysis was used for correlation analysis. A receiver operating characteristic (ROC) curve was used to analyze the diagnostic efficiency and the optimal cut-off value. Differences were considered statistically significant when the $\mathrm{P}$ value $<0.05$.

\section{Results}

\section{Demographic characteristics}

There was no significant difference in gender, onset of day age, rate of cesarean section, gestational age, birth weight, and Apgar score between the observation group and the control group $(\mathrm{P}>0.05)$. There was no significant difference in the incidence of the level of creatinine on the first day and AKI during hospitalization between the 2 groups $(\mathrm{P}>0.05)$. The platelet count in the observation group was 
Table 1 Comparison of general data between the Observation Group and the Control Group

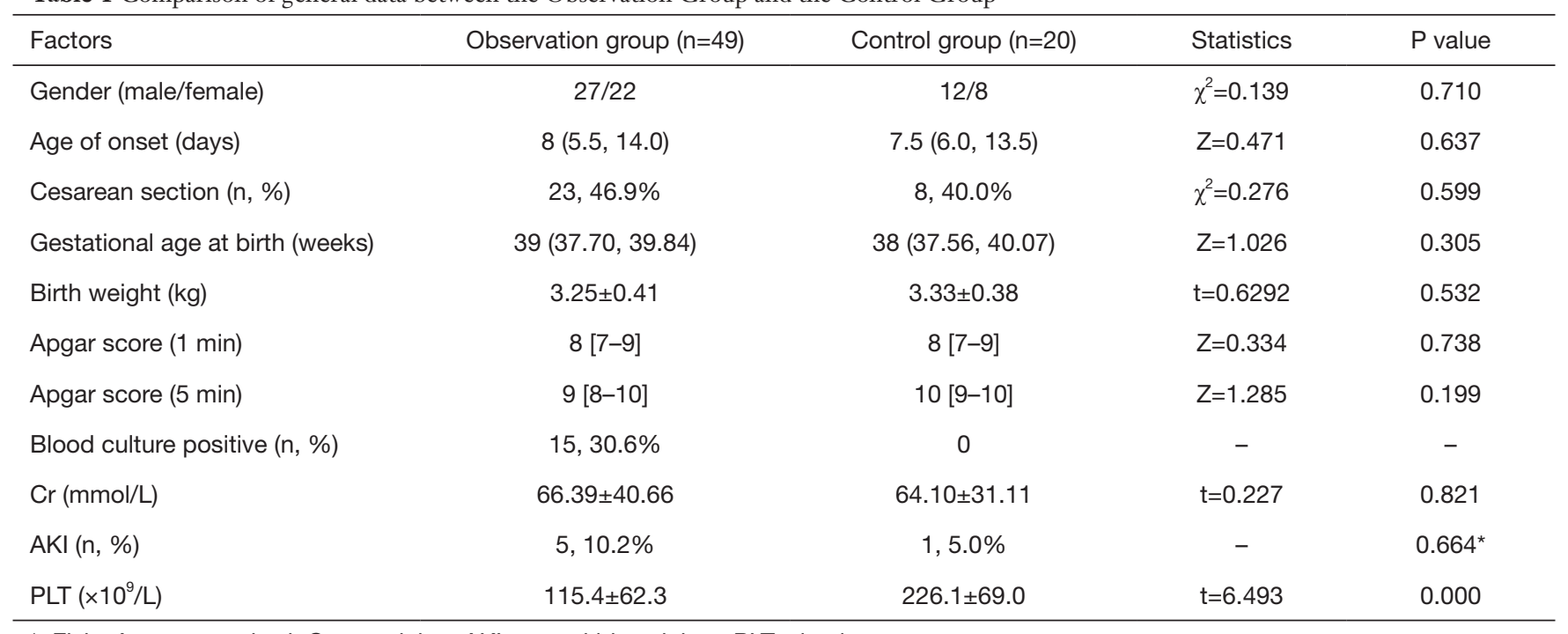

*, Fisher's exact method. Cr, creatinine; AKI, acute kidney injury; PLT, platelet.

Table 2 Changes of sNGAL, CRP, and PCT in both groups

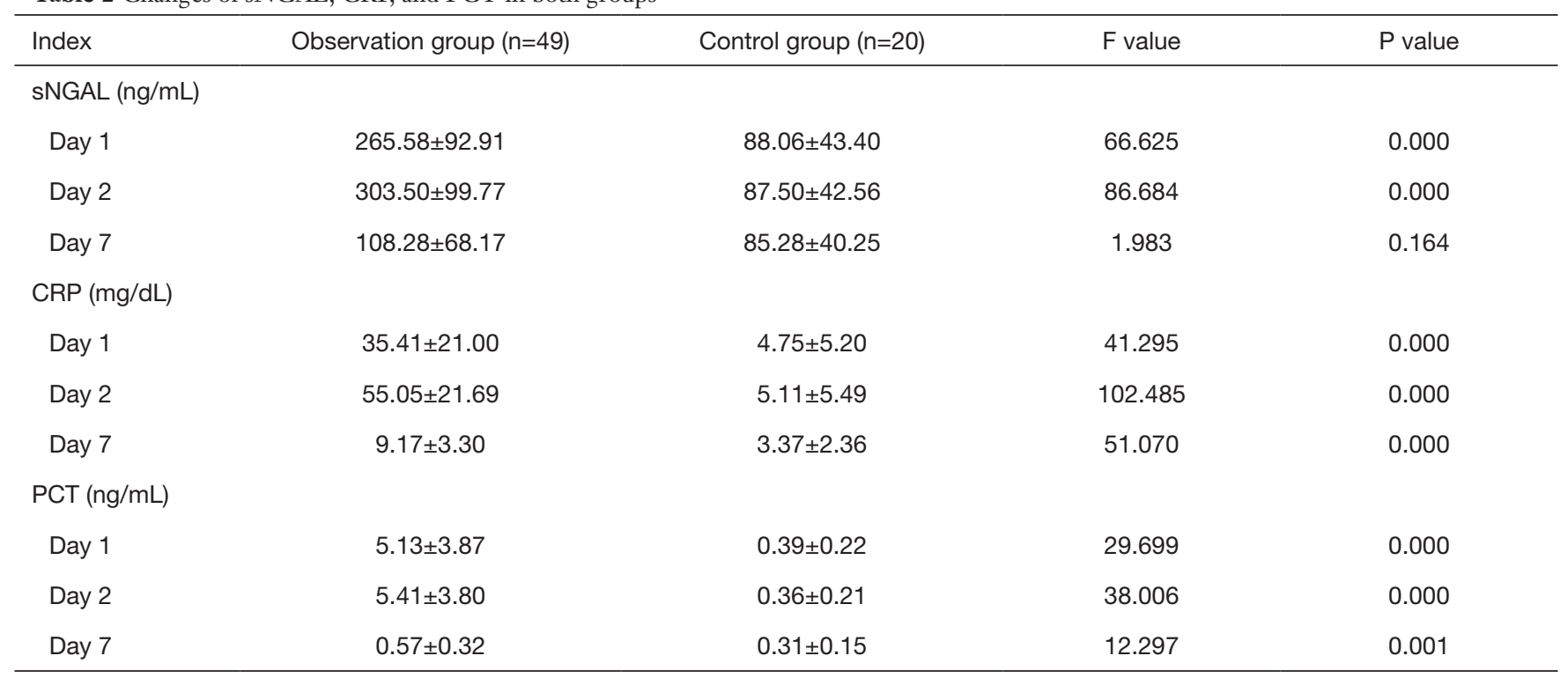

sNGAL, serum neutrophil-gelatinase associated lipocalin; CRP, C-reactive protein; PCT, procalcitonin

lower than that in the control group, and the difference was statistically significant $(\mathrm{P}<0.05)$ (Table 1).

\section{Serum NGAL, CRP, and PCT dynamic change in sepsis}

According to ANOVA of repeated measurement data, the levels of sNGAL, PCT, and CRP in the observation group were all higher than those in the control group at NICU admission on day 1 and day 2, with statistical significance $(\mathrm{P}<0.05)$. There was a trend of change with time of the level of sNGAL. The sNGAL concentration in the observation group began to increase on day 1 , increased more obviously on day 2 , and decreased significantly on day 7 . There was significant difference in the sNGAL concentration on day 1 and day 2 between the observation group and the control $(\mathrm{P}<0.05)$ (Table 2). 


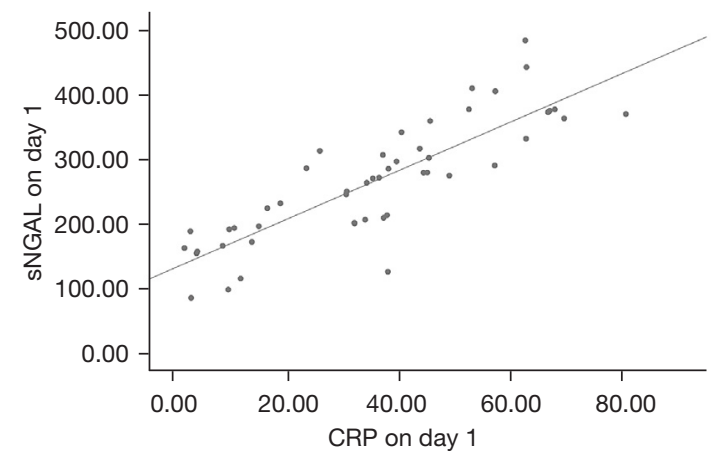

Figure 1 Scatter diagram of correlation analysis between sNGAL and CRP on Day 1. sNGAL, serum neutrophil-gelatinase associated lipocalin; CRP, C-reactive protein.

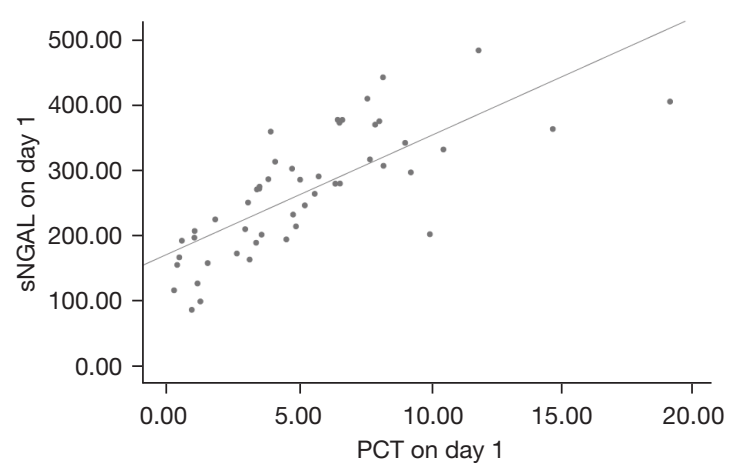

Figure 2 Scatter diagram of correlation analysis between sNGAL and PCT on Day 1. sNGAL, serum neutrophil-gelatinase associated lipocalin; PCT, procalcitonin.

Table 3 ROC analysis of sNGAL, CRP, and PCT on day 1

\begin{tabular}{lccccc}
\hline Index & Cutoff & Sensitivity & Specificity & AUC & $0.95 \%$ Cl \\
\hline sNGAL & $147.5 \mathrm{ng} / \mathrm{mL}$ & $91.8 \%$ & $90.0 \%$ & 0.964 & 0.925 \\
CRP & $9.35 \mathrm{mg} / \mathrm{dL}$ & $85.7 \%$ & $90.0 \%$ & 9.000 & $0.866-0.984$ \\
PCT & $0.73 \mathrm{ng} / \mathrm{mL}$ & $91.8 \%$ & $95.0 \%$ & 0.940 & $0.881-0.998$ \\
\hline
\end{tabular}

ROC, receiver operating characteristic; sNGAL, serum neutrophil-gelatinase associated lipocalin; CRP, C-reactive protein; PCT, procalcitonin; AUC, area under the curve; $\mathrm{Cl}$, confidence interval.

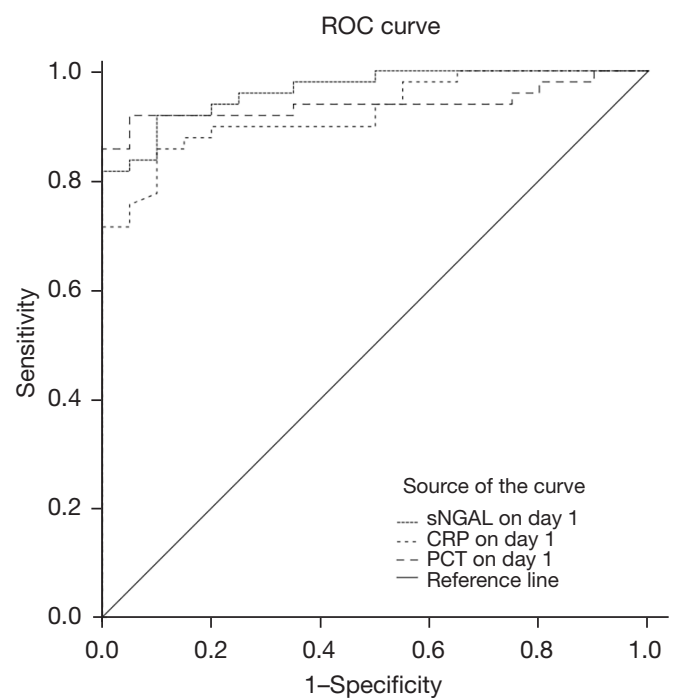

Figure 3 ROC curve of NGAL, CRP, and PCT on day 1. sNGAL, serum neutrophil-gelatinase associated lipocalin; CRP, C-reactive protein; PCT, procalcitonin.

\section{Correlation analysis of sNGAL with CRP and PCT on day 1}

There was a positive correlation between sNGAL and CRP in the observation group on day 1, Pearson's correlation coefficient $=0.845(\mathrm{P}=0.000)$, and the fitting index of the linear regression equation $\mathrm{R}^{2}=0.714$ (Figure 1). There was a positive correlation between sNGAL and PCT in the observation group on day 1, Pearson's correlation coefficient $=0.749(\mathrm{P}=0.000)$, and the fitting index of the linear regression equation $\mathrm{R}^{2}=0.561$ (Figure 2 ).

\section{ROC curve analysis}

The ROC curve was used to evaluate the value of sNGAL, CRP, and PCT in the diagnosis of neonatal sepsis on day 1 (Table 3, Figure 3).

\section{Discussion}

The NGAL is a lipocalin superfamily member, which was 
first discovered and named by Lars Kjeldsen in 1993 (12). Normally, NGAL is released from a small amount of neutrophils in bone marrow while under pathological conditions, and it is also upregulated and released by a variety of other cells, such as activated neutrophils, macrophages, and non-hematopoietic epithelial cells $(13,14)$. Meanwhile NGAL is a novel marker of AKI $(15,16)$, and it has been identified as a highly sensitive and nonspecific predictor of AKI injury in pediatric septic shock (17). In recent years, more attention has been paid not only to AKI, but also the field of sepsis. As an acute secretory protein with bacteriostatic properties, NGAL is released from activated neutrophils in response to bacterial infections. It also can prevent the deterioration of infection by inhibiting the intake of iron of bacteria (18). Katagiri et al. found that the concentrations of sNGAL in patients with sepsis were significantly higher than those in control group participants, suggesting that NGAL has a predictive effect on the early diagnosis of sepsis (19). In our study, we found that the level of sNGAL was significantly elevated in the early stage of neonatal late-onset sepsis. The differences between the observation group and the control group were significant on day 1 and day $2(\mathrm{P}<0.05)$, which indicates that $\mathrm{sNGAL}$ is useful in the early diagnose of neonatal bacterial infection. Jonsson et al. found that septic patients in the ICU showed a significant decrease in NGAL and PCT levels on the first 2 days following empiric antibiotic treatment, which indicates that NGAL may serve as a biomarker to guide downgrading antibiotic therapy in the future (20). We also found that the level of sNGAL decreased obviously on day 7 with the improvement of the diseases, which indicated that sNGAL may have the value of predicting the prognosis and guiding the use of the antibiotics. Albert et al. found that the dynamic changes in SNGAL could predict mortality in patients with severe sepsis (21). It was found that the level of sNGAL was elevated more obviously in neonatal sepsis with positive blood culture (10). We will conduct a subsequent study regarding outcome predictors in our future work.

Smertka et al. found that the level of NGAL in the urine and blood is significantly increased in neonatal sepsis with AKI, which indicates that NGAL is not only a biomarker of sepsis, but also the predictor of AKI (10). In our study, we found that the rate of AKI in the observation group was $10.2 \%$, slightly higher than that in the control group (5.0\%), but the difference was not statistically significant, indicating that the association between sNGAL and AKI was present in our study. In this study, there were only 5 cases of AKI in the observational group which made it impossible to conduct subgroup analysis and to certify whether sNGAL had a predictive effect of AKI in the neonatal sepsis.

Currently, PCT and CRP are regarded as the common biomarkers in the diagnosis of neonatal sepsis (6). In some studies, PCT has been evaluated as a marker for diagnosing neonatal sepsis with higher sensitivity and specificity than CRP (6). In our study, we found that the level of sNGAL, CRP, and PCT of the observation group was higher than that in the control group on day 1 and day 2, which indicated the activation of the inflammation in the early stage of neonatal sepsis. Through correlation analysis, we found that the level of sNGAL was significantly correlated with the level of CRP and PCT on day 1 and day 2, which certified that sNGAL was well correlated with these classical biomarkers such as CRP and PCT in the diagnosis of neonatal sepsis.

The ROC curve was used to compare the efficiency of various biomarkers in the diagnosis of neonatal sepsis. The results of ROC indicated that the respective sensitivity of sNGAL, CRP, and PCT was $91.8 \%, 85.7 \%$, and $91.8 \%$, the respective specificity was $90 \%, 90 \%$, and $95 \%$, and the areas under the curve were $0.964,0.925$, and 0.940 , respectively. The above results showed that the ability of sNGAL in the diagnosis of neonatal sepsis is better than CRP, while the diagnostic efficacy of sNGAL and PCT is similar. Therefore, we believe that sNGAL can be used as a predictive and diagnostic index of neonatal sepsis and to monitor the improvement of the disease. Researchers within China and internationally have been seeking infection-related markers with high sensitivity, specificity, positive predictive value, and negative predictive value to reliably distinguish sepsis at an earlier level and instruct the antibiotic therapy. However, none of the current biomarkers are sufficiently independently accurate for diagnosis or prognosis in sepsis until now. Therefore, combined detection of multiple infectious biomarkers, to a certain extent, is more beneficial to the early diagnosis of neonatal sepsis.

\section{Conclusions}

In our study, we found that sNGAL was significantly elevated in the early stage of late-onset neonatal sepsis and obviously decreased with the conditions improvement. At the same time, sNGAL had a good correlation with CRP and PCT. The utilities of sNGAL may be applied for the early diagnosis, monitoring the outcome, and directing the therapy of neonatal late-onset sepsis. Some limitations of 
this study should be considered. The participants were all from a single center and the sample size was small. The selective bias was inevitable. In future research, we will further expand the sample size and conduct further research from the perspective of outcome prediction.

\section{Acknowledgments}

Funding: The study was supported by Natural Science Research Project of Nantong Science and Technology Bureau (JCZ19031, MSZ20078).

\section{Footnote}

Reporting Checklist: The authors have completed the STARD reporting checklist. Available at https://tp.amegroups.com/ article/view/10.21037/tp-21-587/rc

Data Sharing Statement: Available at https://tp.amegroups. com/article/view/10.21037/tp-21-587/dss

Conflicts of Interest: All authors have completed the ICMJE uniform disclosure form (available at https://tp.amegroups. com/article/view/10.21037/tp-21-587/coif). The authors have no conflicts of interest to declare.

Ethical Statement: The authors are accountable for all aspects of the work in ensuring that questions related to the accuracy or integrity of any part of the work are appropriately investigated and resolved. As per instructions of the Ethics Committee of the Affiliated Hospital of Nantong University, informed consent was provided by the guardians of patients before commencement of the study. The study was approved by the Ethics Committee of the Affiliated Hospital of Nantong University (2021-K141-01), and was conducted in accordance with the Declaration of Helsinki (as revised in 2013).

Open Access Statement: This is an Open Access article distributed in accordance with the Creative Commons Attribution-NonCommercial-NoDerivs 4.0 International License (CC BY-NC-ND 4.0), which permits the noncommercial replication and distribution of the article with the strict proviso that no changes or edits are made and the original work is properly cited (including links to both the formal publication through the relevant DOI and the license). See: https://creativecommons.org/ licenses/by-nc-nd/4.0/.

\section{References}

1. Kim F, Polin RA, Hooven TA. Neonatal sepsis. BMJ 2020;371:m3672.

2. Shane AL, Sánchez PJ, Stoll BJ. Neonatal sepsis. Lancet 2017;390:1770-80.

3. Popescu CR, Cavanagh MMM, Tembo B, et al. Neonatal sepsis in low-income countries: epidemiology, diagnosis and prevention. Expert Rev Anti Infect Ther 2020;18:443-52.

4. Carbone F, Montecucco F, Sahebkar A. Current and emerging treatments for neonatal sepsis. Expert Opin Pharmacother 2020;21:549-56.

5. Esposito $\mathrm{S}$, Principi N. Adjunctive therapy to treat neonatal sepsis. Expert Rev Clin Pharmacol 2020;13:65-73.

6. Ruan L, Chen GY, Liu Z, et al. The combination of procalcitonin and C-reactive protein or presepsin alone improves the accuracy of diagnosis of neonatal sepsis: a meta-analysis and systematic review. Crit Care 2018;22:316.

7. Benitz WE, Achten NB. Technical assessment of the neonatal early-onset sepsis risk calculator. Lancet Infect Dis 2021;21:e134-40.

8. Stoll BJ, Puopolo KM, Hansen NI, et al. Early-Onset Neonatal Sepsis 2015 to 2017, the Rise of Escherichia coli, and the Need for Novel Prevention Strategies. JAMA Pediatr 2020;174:e200593.

9. Hashem HE, El Masry SA, Mokhtar AM, et al. Valuable Role of Neutrophil CD64 and Highly Sensitive CRP Biomarkers for Diagnostic, Monitoring, and Prognostic Evaluations of Sepsis Patients in Neonatal ICUs. Biomed Res Int 2020;2020:6214363.

10. Smertka M, Wroblewska J, Suchojad A, et al. Serum and urinary NGAL in septic newborns. Biomed Res Int 2014;2014:717318.

11. Hao YX, Yu JL. Cut-off value of white blood cell count in the diagnosis of early-onset sepsis in neonates. Zhongguo Dang Dai Er Ke Za Zhi 2020;22:1159-63.

12. Kjeldsen L, Johnsen AH, Sengeløv H, et al. Isolation and primary structure of NGAL, a novel protein associated with human neutrophil gelatinase. J Biol Chem 1993;268:10425-32.

13. Yin X, Huo Y, Liu L, et al. Serum Levels and Placental Expression of NGAL in Gestational Diabetes Mellitus. Int J Endocrinol 2020;2020:8760563.

14. Xiang D, Wang X, Liu P, et al. Increased NGAL level associated with iron store in chronic kidney disease with anemia. Clin Exp Med 2018;18:563-8.

15. Wei B, Tian T, Liu YG. IL-10 Combined with NGAL 
Has Diagnostic Value for AECOPD Combined with AKI. Int J Chron Obstruct Pulmon Dis 2020;15:637-44.

16. Lee SA, Noel S, Kurzhagen JT, et al. CD4+ T CellDerived NGAL Modifies the Outcome of Ischemic Acute Kidney Injury. J Immunol 2020;204:586-95.

17. Peerapornratana S, Priyanka P, Wang S, et al. SepsisAssociated Acute Kidney Disease. Kidney Int Rep 2020;5:839-50.

18. Goetz DH, Holmes MA, Borregaard N, et al. The neutrophil lipocalin NGAL is a bacteriostatic agent that interferes with siderophore-mediated iron acquisition. Mol Cell 2002;10:1033-43.

19. Katagiri D, Doi K, Matsubara T, et al. New biomarker panel of plasma neutrophil gelatinase-associated lipocalin

Cite this article as: Cai Q, Zhang X, Shen L, Wang T. Clinical application value of serum neutrophil gelatinase-associated lipocalin in neonatal sepsis. Transl Pediatr 2022;11(1):120-126. doi: $10.21037 / \mathrm{tp}-21-587$ and endotoxin activity assay for detecting sepsis in acute kidney injury. J Crit Care 2013;28:564-70.

20. Jonsson N, Gille-Johnson P, Martling CR, et al. Performance of plasma measurement of neutrophil gelatinase-associated lipocalin as a biomarker of bacterial infections in the intensive care unit. J Crit Care 2019;53:264-70.

21. Albert C, Zapf A, Haase M, et al. Neutrophil GelatinaseAssociated Lipocalin Measured on Clinical Laboratory Platforms for the Prediction of Acute Kidney Injury and the Associated Need for Dialysis Therapy: A Systematic Review and Meta-analysis. Am J Kidney Dis 2020;76:826841.e1. 